\title{
Lifestyle changes in arterial hypertension - an important objective of cardiovascular rehabilitation. The
} role of physical exercise

\author{
POP Dana ${ }^{1,2}$, DĂDÂRLAT-POP Alexandra ${ }^{1,3}$, CISMARU Gabriel ${ }^{1,2}$, \\ ZDRENGHEA Dumitru ${ }^{1,2}$, CALOIAN Bogdan ${ }^{1,2}$
}

Corresponding author: Alexandra Dădârlat-Pop, E-mail: dadarlat.alexandra@yahoo.ro

1. Internal Medicine Department, "Iuliu Hatieganu” University of Medicine and Pharmacy, Cluj-Napoca, Romania 2. Rehabilitation Hospital, Cluj-Napoca, Romania 3. "N. Stancioiu" Heart Institute, Cluj-Napoca, Romania

\begin{abstract}
Cardiovascular rehabilitation includes lifestyle changing measures, secondary drug prevention and physical training programs. Identifying and addressing cardiovascular risk factors, among which arterial hypertension, is an important objective of cardiovascular rehabilitation. Patients with arterial hypertension should be initially assigned to a cardiovascular risk class. Alongside drug therapy, a healthy diet and physical activity play an important role in the control of blood pressure values
\end{abstract}

Keywords: arterial hypertension, physical exercise,

\section{Introduction}

Cardiovascular rehabilitation includes lifestyle changing measures, secondary drug prevention and physical training programs. Identifying and addressing cardiovascular risk factors, among which arterial hypertension (HTN), is an important objective of cardiovascular rehabilitation. Patients with arterial hypertension should be initially assigned to a low, moderate, high or very high cardiovascular risk class. Risk stratification is achieved depending on the degree of HTN, hypertension-mediated organ damage, disease stage, presence of other risk factors or comorbidities $(1,2)$. However, regardless of this stratification, HTN requires in the first place the adoption of lifestyle changing measures (1). Without any doubt, a healthy diet plays an extremely important role. In HTN, the DASH diet (rich in fruit, vegetables, integral cereals and low fat dairy products, with a reduced saturated and total fat content) has proved to be highly efficient, leading to decreases in systolic blood pressure by $11 \mathrm{mmHg}$ on an average (3). Salt intake should be less than 5 grams/day (3) and sodium intake should be less than $2 \mathrm{mg} /$ day, ideally less than $1.5 \mathrm{mg} /$ day (3). At the same time, a diet rich in potassium, 3500-5000 mg/day, contributes to decreasing blood pressure values (3). Also, maintaining a normal weight is important (3).

Physical activity is also part of the recommendations included in lifestyle changes for hypertensive patients $(1,3)$. Certainly, physical activity is important for maintaining a good health status for all categories of individuals, for the population in general (4). Exercise performed regularly has important cardioprotective effects (5). These can be grouped into:

- antiatherosclerotic effects: increase of insulin sensitivity, decrease of inflammation, decrease of platelet aggregability, decrease of fibrinogen, increase of fibrinolysis, increase of nitric oxide release, improvement of endothelial function

- possible antiarrhythmic effects: increase of vagal tone and decrease of sympathetic activity.

At the same time, there is evidence that physical activity reduces lipid fractions, i.e. LDL cholesterol, triglycerides and cholesterol, with a significant increase in HDL cholesterol
(6). Physical training programs performed constantly contribute to decreasing the levels of some inflammation markers such as C-reactive protein (7). In the case of diabetic patients, exercise can decrease insulin resistance (7). At the same time, it improves arterial flow-mediated dilatation (7). A recently published meta-analysis demonstrates the fact that constant physical activity improves endothelial dysfunction (8).

Constant, long-duration physical exercise plays a role in both preventing the development of HTN and decreasing blood pressure values in the case of patients diagnosed with HTN. HTN prevention is due to the decrease of vascular resistance, arterial stiffness, oxidative stress, inflammation, synaptic activation, the renin-angiotensin system (9). On the other hand, there is an improvement in endothelial function, insulin sensitivity, angiogenesis, parasympathetic activity and renal function (9). In fact, in a study published in 2017 in USA, it was demonstrated that moderate vigorous physical activity or sports activities led to a reduction of the risk of HTN (10). Regarding the decrease of blood pressure values in patients with HTN, various molecular and biochemical mechanisms are incriminated. The decrease of sympathetic activity, with a significant $29 \%$ reduction of norepinephrine release and a $20 \%$ reduction of the renin level, represents one of the most important actions of constant physical activity (11). It also has effects on contractility and calcium handling, cardiomyocyte turnover and microRNAs (11). One of the most important complications of HTN is left ventricular hypertrophy. Over the course of time, various studies have been published, the majority of which have demonstrated that regular physical activity may cause the regression of this hypertrophy. A 2020 meta-analysis shows that along with antihypertensive drug therapy, physical activity leads to a reduction of left ventricular mass (12).

One of the target organs frequently affected by arterial hypertension is the kidney. In the early, asymptomatic phases of kidney injury, microalbuminuria occurs, but when the blood pressure values are maintained high or when additional 
risk factors such as diabetes mellitus are present, patients evolve towards chronic kidney disease and renal failure (13). The prevalence of cardiovascular diseases in this category of patients is increased compared to the general population; these have a higher frequency of atherosclerotic lesions, calcific valvular lesions, as well as rhythm disorders favored by electrolytic imbalances.

Their exercise capacity can be reduced because of secondary anemia of renal cause, uremic myopathy and polyneuropathy, hydroelectrolytic and acid-base imbalances. Patients after kidney transplantation are also affected by the physical inactivity involved by the procedure and by immunosuppressive treatment (14).

Dietary, lifestyle changes, as well as inclusion in cardiovascular rehabilitation programs are measures that can effectively complement the pharmacological treatment of patients with arterial hypertension and chronic kidney disease, significantly slowing the evolution towards endstage chronic renal failure that requires chronic hemodialysis or kidney transplantation (15).

Regarding diet, phosphate rich foods (milk, eggs, meat), calcium and vitamin D supplements are recommended, while potassium containing foods (fresh fruit, fruit juice, walnuts) should be avoided (1).

\section{Exercise recommendations in HTN}

Before physical training recommendations, patients should be evaluated not only for blood pressure control, but also for the presence of other cardiovascular risk factors, for target organ involvement. At the same time, an exercise test will be performed, which will assess the way in which blood pressure is controlled during exercise.

It should be highlighted that patients with HTN who performed constant leisure physical activity had a $24 \%$ lower risk (women) and a $27 \%$ lower risk (men) of cardiovascular diseases compared to men and women with reduced physical activity levels (16).

Moderate intensity physical exercise is associated with a decrease of at least $15 \%$ in mortality $(17,18)$.

According to current guidelines, physical activity recommended for cardiovascular patients is of two types.

The first - physical activity counseling - consists of the recommendation to perform constant exercise at least 5 times/week at home or in fitness rooms. This activity is not supervised by specialized personnel, but self-monitoring is recommended, in the first place by monitoring heart rate and the Borg Scale of Perceived Exertion.

The second type of activity is physical training performed only in certain cardiovascular diseases (in general more severe or with a risk of being worsened by excessive exercise). This will be practiced in hospital, in ambulatory rehabilitation centers and, in special situations, at home (telemedicine) and will be supervised by specialized personnel (physiotherapist, nurse), being preceded by an exercise test establishing the parameters and characteristics of physical exercise according to the FITT (frequency, intensity, time and type of exercise) model (19).
In arterial hypertension without other comorbidities, only physical counseling is recommended in general, physical training being recommended for hypertension associated with other cardiovascular disorders which requires supervised rehabilitation. The methodology applied in this situation will be that recommended for the disease concerned, but a good control of blood pressure and its careful monitoring throughout the exercise duration are necessary (19).

Current guidelines recommend for patients with HTN, with controlled blood pressure, at least 30 minutes of moderate intensity dynamic aerobic exercise (walking, jogging, cycling or swimming) on 5-7 days per week $(1,20)$. For additional benefits, in patients with a low risk, a gradual increase in moderate intensity dynamic aerobic exercise to 300 minutes a week or 150 minutes of vigorous intensity aerobic physical activity per week or a combination of these is recommended (18). In the case of adults with a high cardiovascular risk and/or hypertension-mediated organ damage, high-intensity resistance exercise is not recommended (18). At the same time, if systolic blood pressure values are higher than $160 \mathrm{mmHg}$, high-intensity exercise will not be performed until HTN is well controlled (20).

Performing resistance exercises for 2-3 days a week can also be recommended in the case of patients with well controlled blood pressure values, with potential effects in decreasing systolic blood pressure values, on an average by $5 \mathrm{mmHg}(1$, 3 ). However, high-intensity resistance training should not be initiated in the case of persons inexperienced with more moderate resistance exercises, regardless of age, health status or training level.

If individuals with controlled HTN want to practice high performance sport, antihypertensive treatment should consist of administering angiotensin converting enzyme inhibitors, sartans and calcium channel blockers (20). Beta-blockers and diuretics will be avoided (20). At the same time, in the presence of hypertension-mediated organ damage and high cardiovascular risk but well controlled HTN, sports will not be forbidden, except for more intensive power disciplines such as weightlifting, discus/javelin throwing and shotputting (20).

In general, patients with arterial hypertension and chronic kidney disease receive the same rehabilitation recommendations as those with cardiovascular disorders and normal renal function, with a few mentions. Patients included in chronic hemodialysis programs may require an individualized program, with the protection of the area where the arteriovenous fistula is found (avoidance of traumas and exercises that lead to an excessive increase in pressure in the upper limb concerned, measurement of blood pressure in the other arm) and the scheduling of the training sessions on the days when no hemodialysis is performed (19).

In conclusion, a regular physical activity program may contribute in the case of hypertensive patients to decreasing blood pressure values, as well as cardiovascular risk. 


\section{References:}

1. Williams B, Mancia G, Spiering W et al. 2018 ESC/ESH Guidelines for the management of arterial hypertension. Eur Heart J. 2018; 39(33): 3021-3104.

2. Molnar A, Sacui D, Scridon T. Risk Factors Influencing the Surgical Outcome in 138 Consecutive Patients with Infrarenal Aortic Aneurysm: The Cluj-Napoca Cardiovascular Surgery Center Experience CHIRURGIA. 2014;109:223-228.

3. Arnett DK, Blumenthal RS, Albert MA, Buroker AB, Goldberger ZD, Hahn EJ, Himmelfarb CD. 2019 ACC/AHA Guideline on the Primary Prevention of Cardiovascular Disease: A Report of the American College of Cardiology/American Heart Association Task Force on Clinical Practice Guidelines. J Am Coll Cardiol. 2019 ;74(10):1376-1414.

4. Piepoli MF, Hoes AW, Agewall S, Albus C, et al. 2016 European Guidelines on cardiovascular disease prevention in clinical practice: The Sixth Joint Task Force of the European Society of Cardiology and Other Societies on Cardiovascular Disease Prevention in Clinical Practice (constituted by representatives of 10 societies and by invited experts) Developed with the special contribution of the European Association for Cardiovascular Prevention \& Rehabilitation (EACPR). Eur Heart J. 2016; 37(29): 2315-2381.

5. Boden WE, Franklin B, Berra K, Haskell WL, Calfas KJ, Zimmerman FH, Wenger NK. Exercise as a therapeutic intervention in patients with stable ischemic heart disease: an underfilled prescription. Am J Med. 2014;127(10):905-11.

6. Kraus WE, Slentz CA. Exercise training, lipid regulation, and insulin action: a tangled web of cause and effect. Obesity (Silver Spring). 2009;17 Suppl 3:S21-6.

7. Lavie CJ. Church TS, Milani R V, Earnest C P. Impact of Physical Activity, Cardiorespiratory Fitness, and Exercise Training on Markers of Inflammation. Journal of Cardiopulmonary Rehabilitation and Prevention. 2011;31(3):137-145.

8. Lanza GA, Golino M Villano A, Lanza O, Lamendola P, Fusco A, Leggio M. Cardiac Rehabilitation and Endothelial Function. J Clin Med . 2020;9(8):2487.

9. Diaz KM, Shimbo D. Physical activity and the prevention of hypertension.

10. Curr Hypertens Rep. 2013;15(6):659-68

11. Keith M Diaz, John N Booth III, Samantha R Seals, Marwah Abdalla, Patricia M Dubbert, Mario Sims, Joseph A Ladapo, Nicole Redmond, Paul Muntner, Daichi Shimbo. Physical Activity and Incident Hypertension in African Americans. Hypertension. 2017; 69(3): 421-427.

12. Hegde SM, Solomon SD. Influence of Physical Activity on Hypertension and Cardiac Structure and Function. Curr Hypertens Rep. 2015 ; 17(10): 77.

13. Tomaz de Castro QJ, Corrêa Tomaz FS, Watai PY, GrabeGuimarães A. Physical Exercise Combined with Antihypertensive Drug Therapy on Left Ventricular Hypertrophy: Systematic Review and Meta-Analysis. High Blood Press Cardiovasc Prev. 2020;27(6):493-503.

14. Muxfeldt, E.S., de Souza, F., Margallo, V.S. et al. Cardiovascular and Renal Complications in Patients with Resistant Hypertension. Curr Hypertens Rep 16, 471 (2014). https://doi.org/10.1007/s11906-014-0471-7.

15. Bakris GL, Williams M, Dworkin L, Elliott WJ, Epstein M, Toto R, Tuttle K, Douglas J, Hsueh W, Sowers J. Preserving renal function in adults with hypertension and diabetes: A consensus approach, American Journal of Kidney Diseases, Volume 36, Issue 3 2 2000, Pages 646-661,ISSN 0272-6386 https://doi.org/10.1053/ajkd.2000.16225.
16. Zoccali, C., Mallamaci, F. Uric Acid, Hypertension, and Cardiovascular and Renal Complications. Curr Hypertens Rep 15, 531-537 (2013). https://doi.org/10.1007/s11906-013-0391-y

17. Li J, Siegrist J. Physical activity and risk of cardiovascular diseasea meta-analysis of prospective cohort studies. Int $\mathrm{J}$ Environ Res Public Health 2012; 9:391-407.

18. Leitzmann M.F, Park, Y; Blair A, Ballard-Barbash R, Mouw T, Hollenbeck A.R, Schatzkin A. Physical activity recommendations and decreased risk of mortality. Arch Intern Med 2007; 167:24532460 ;

19. Rossi A, Dikareva A, Bacon S.L, Daskalopoulou S.S. The impact of physical activity on mortality in patients with high blood pressure: a systematic review. J Hypertens 2012;30:1277-1288.

20. Ambrosetti M, Abreu A, Corrà U, et al. Secondary prevention through comprehensive cardiovascular rehabilitation: From knowledge to implementation. 2020 update. A position paper from the Secondary Prevention and Rehabilitation Section of the European Association of Preventive Cardiology [published online ahead of print, 2020 Mar 30]. Eur J Prev Cardiol. 2020;2047487320913379. doi:10.1177/2047487320913379.

21. Pelliccia A, Sharma S, Gati S, Bäck M, Börjesson M, Caselli S et al. 2020 ESC Guidelines on sports cardiology and exercise in patients with cardiovascular disease. Eur Heart J. 2020 Aug 29; ehaa605. doi: 10.1093/eurheartj/ehaa605.

22. Amarowicz . Tannins: The new natural antioxidants? European Journal of Lipid Science and Technology, 2007, 109(6): 549-551.. 